\title{
Detection of Hepatitis B Virus-Like Nucleotide Sequences in Liver Samples from Murine Rodents and Asian House Shrews
}

\author{
Wen-Qiao He, Xue-Jiao Chen, Yu-Qi Wen, Yong-Zhi Li, Huan He, and Qing Chen
}

\begin{abstract}
In recent years, hepatitis B virus (HBV) has been detected in some species of animals. In this study, we found HBV-like nucleotide sequences in murine rodents and Asian house shrews (Suncus murinus) collected in China. A total of 801 animals were trapped. We found that $0.48 \%(3 / 624)$ of the murine rodents and $1.69 \%(3 / 177)$ of Asian house shrews were positive for HBV-like DNA. Detection of HBV-like DNA in brown rats (Rattus norvegicus), rice-field rat (Rattus losea), and Asian house shrews indicated that these species of animals might be hosts for HBV. However, none of the HBV-like DNA-positive animals was additionally positive for antiHBV antibodies or hepatitis B surface antigen. A 585 bp nucleic acid sequence, mapping to a hepadnavirus, was extracted from rice-field rat, and bores strong resemblance to human HBV genotype B sequences. Further research is required to investigate the hepadnaviruses within the murine rodent and Asian house shrew populations to uncover the origin and zoonotic potential of HBV.
\end{abstract}

Keywords: Asian house shrews, hepadnavirus, murine rodents, prevalence, zoonotic potential

\section{Introduction}

$\mathbf{H}$ EPATITIS B VIRUs (HBV), the causative agent of hepatitis $\mathrm{B}$, is a double-stranded DNA virus belonging to the genus Orthohepadnavirus in the family Hepadnaviridae (Locarnini et al. 2013). Although there is a preventative vaccine against HBV, it still remains a serious global health problem. In China, HBV caused 256,000 deaths in 2010 and brought significant burden in some regions, such as Guangdong (11.1\%), Hunan $(7.3 \%)$, Fujian (12-13\%), and Yunnan (8.4\%) provinces (Xiao et al. 2012, Xia et al. 2015, Kang et al. 2017).

HBV has been found in some species of animals; however, the host range of $\mathrm{HBV}$ remains elusive (Locarnini et al. 2013). With the discovery of the tent-making bat HBV, HBV is now viewed as a potentially zoonotic pathogen (Drexler et al. 2013).

To date, HBV or HBV-like viruses have not been found in murine rodents or shrews (Van Nguyen et al. 2018). In this study, we investigated HBV in these species of animals.

\section{Methods}

Samples

Between 2014 and 2017, small animals were trapped in China (Supplementary Table S1 and Supplementary Fig. S1). Serum and liver tissue samples were collected and stored at $-80^{\circ} \mathrm{C}$ until testing. The species of animals were identified by sequencing of cytochrome B (cytB) gene (Arai et al. 2008).

\section{Extraction of nucleic acid and detection of $\mathrm{HBV}$}

The MiniBEST Viral RNA/DNA Extraction Kit (TaKaRa, Japan) was used to extract total RNA and DNA from liver tissue samples. A broadly reactive and highly sensitive nested PCR assay was used to detect HBV DNA (Drexler et al. 2013).

The positive samples were sent to the Beijing Genomics Institute for sequencing. 


\section{Amplification of HBV genome}

Based on six human HBV sequences (GenBank accession numbers: KM392074, FJ562311, AY217369, AF121250, KC774399, DQ993706), we designed primers to amplify the viral genome (Supplementary Table S2). Lasergene SeqMan software (DNASTAR, Madison) was used to assemble the sequences.

\section{Phylogenetic analysis}

Sequences obtained in this study were aligned using the ClustalW multiple sequence alignment program in MEGA (version 7.0; Oxford Molecular Ltd., UK). Phylogenetic tree was constructed using the neighbor-joining method, with 1000 bootstrap replicates.

The sequences identity was calculated using Sequence Identity Matrix program in BioEdit (Version 7.2.5).

\section{Detection of anti-HBV antibodies and $H B V$ surface antigen}

Serum samples and supernatants of liver tissue samples from HBV-like DNA-positive animals were assayed for the presence of HBV e antibody, HBV surface antibody, HBV core antibody, and HBV surface antigen (HBsAg) by using enzyme-linked immunosorbent assay (ELISA) kits (CUSABIO, China).

\section{Ethics guidelines}

The protocol for this study was approved by the ethics committee of the Institutional Animal Care and Use Committee of Southern Medical University.

\section{Results and Discussion}

A total of 801 animals were trapped. HBV-like DNA was detected in $0.48 \%$ of murine rodents (3/624) and $1.69 \%$ of the Asian house shrews (Suncus murinus) (3/177) (Supplementary Table S1). The HBV-like DNA-positive Asian house shrews were trapped in the same site in Guangzhou, but not at the same time. Discovery of HBV-like DNA in brown rats (Rattus norvegicus), rice-field rat (Rattus losea), and Asian house shrews indicated that these species of animals might be hosts for HBV, and the host range of HBV could be wider than previously thought. The low detection rates indicated either a spillover infection or a low prevalence of HBV in these populations. More sensitive detection techniques are required in the future investigation.

The sequences, about $100 \mathrm{bp}$ in length from HBV-like DNA-positive animals, were obtained from the nested PCR assay, and showed high degree of similarity with human HBV sequence (Supplementary Table S3). We only obtained a 585 bp sequence (XM24, GenBank accession number: MK158228), which is closely matched to human HBV sequence at both nucleotide and amino acid levels (Supplementary Table S4), from a rice-field rat with one pair of the designed primers (HBV-F25, HBV-R414, and HBV-R421; Supplementary Table S2) and the primers reported in a previous study (Van Nguyen et al. 2018). Amplification was failed in the other five HBV-like DNA-positive samples. The remaining designed primers failed in amplifying full-length genome, this may be attributed to the diversity of nucleic acid regions that we had not amplified.

HBV is diverse between different species of animals and it was traditionally considered to be a host-specific virus (Drexler et al. 2013). However, the discovery of human

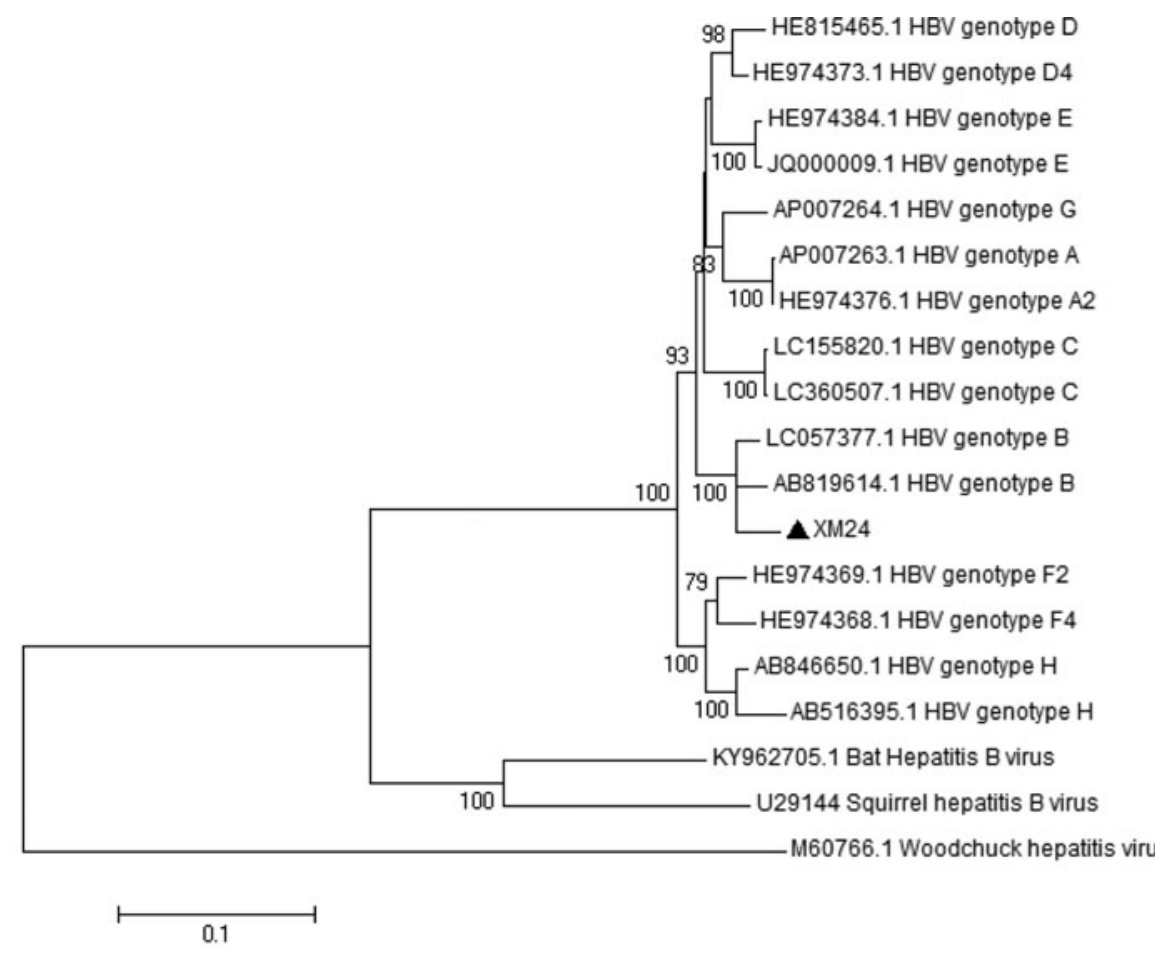

FIG. 1. Phylogenetic tree constructed using the neighbor-joining method, based on partial nucleotide sequence $(585 \mathrm{bp})$ alignment of 19 HBV sequences. Eighteen representative HBV sequences derived from humans, bat, woodchuck, and squirrel. HBV, hepatitis B virus. 
HBV-like DNA in murine rodents and Asian house shrews highlights the zoonotic potential of HBV. To ensure the credibility, phylogenetic analysis was only performed based on the $585 \mathrm{bp}$ sequence. The sequence clustered with human HBV genotype B (Fig. 1). HBV genotype B is common in Asia (Rasche et al. 2016). If there are novel hepadnaviruses from murine rodents and Asian house shrews, and the HBVlike viruses shared similarities with the human HBV prevalent in the same geographical region, one may speculate that they might share a common viral ancestor. However, without full-length HBV genome, we cannot conclude whether the hepadnaviruses in these animals were of human origin due to pathogen spillover or derived from rats or shrews.

None of the HBV-like DNA-positive animals was positive for anti-HBV antibodies or HBsAg, it might be explained by the seronegative occult HBV infection (Makvandi 2016).

Although this study provides new insights into the host range and origin of HBV, the authors acknowledge its limitations. For instance, attempts at the full-length hepadnaviral genome amplification failed. Furthermore, without any suitable HBV cell culture models, we did not perform cell culture experiment. We endeavor to conduct further experiments, with the aim of obtaining the full-length sequence of the virus, in the future.

\section{Conclusion}

HBV-like DNA was detected in brown rats, rice-field rat, and Asian house shrews. A 585 bp sequence, sourced from rice-field rat, showed high similarity to human $\mathrm{HBV}$ sequences. More research is needed to investigate the nature of hepadnaviruses in these animals, be they of rodent or human origin. The zoonotic potential of HBV also merits further investigation.

\section{Acknowledgments}

We are grateful to Xue-Shan Zhong, Shao-Wei Chen, Xue-Yan Zheng, Shu-Juan Ma, Li-Na Jiang, Jin Ge, Ming Qiu, Shu-Ting Huo, Xue-Mei Ke, Wen Zhou, Xing Li, Yun Mo, Yi-Quan Xiong, Ming-Ji Cheng, Yan-Xia Chen, MinYi Zhang, and Fang-fei You for their participation in sample collection. This study was supported by the National Natural Science Foundation of China (grant no. 81373051).

\section{Author Disclosure Statement}

No conflicting financial interests exist.

\section{Supplementary Material}

Supplementary Figure S1

Supplementary Table S1

Supplementary Table S2

Supplementary Table S3

Supplementary Table S4

\section{References}

Arai S, Bennett SN, Sumibcay L, Cook JA, et al. Phylogenetically distinct hantaviruses in the masked shrew (Sorex cinereus) and dusky shrew (Sorex monticolus) in the United States. Am J Trop Med Hyg 2008; 78:348-351.

Drexler JF, Geipel A, Konig A, Corman VM, et al. Bats carry pathogenic hepadnaviruses antigenically related to hepatitis B virus and capable of infecting human hepatocytes. Proc Natl Acad Sci U S A 2013; 110:16151-16156.

Kang WY, Tian ZY, Li QF, Zhang LF, et al. [Sero-epidemiological survey on hepatitis B virus in Yunnan province, between 2006 and 2014]. Zhonghua Liu Xing Bing Xue Za Zhi 2017; 38:518-521.

Locarnini S, Littlejohn M, Aziz MN, Yuen L. Possible origins and evolution of the hepatitis B virus (HBV). Semin Cancer Biol 2013; 23:561-575.

Makvandi M. Update on occult hepatitis B virus infection. World J Gastroenterol 2016; 22:8720-8734.

Rasche A, Souza B, Drexler JF. Bat hepadnaviruses and the origins of primate hepatitis B viruses. Curr Opin Virol 2016; 16:86-94.

Van Nguyen D, Van Nguyen C, Bonsall D, Ngo TT, et al. Detection and characterization of homologues of human hepatitis viruses and pegiviruses in rodents and bats in Vietnam. Viruses 2018; 10:E102.

Xia W, Dai D, Song L, Yan J. Prevalence of hepatitis B virus surface antigen (HBsAg) and antibody to HBsAg among 1-59 year olds, Hunan Province. Chin J Vaccine Immunization 2015; 21:373-377+427.

Xiao J, Zhang J, Wu C, Shao X, et al. Impact of hepatitis B vaccination among children in Guangdong Province, China. Int J Infect Dis 2012; 16:e692-e696.

Address correspondence to: Qing Chen Provincial Key Laboratory of Tropical Disease Research Department of Epidemiology School of Public Health Southern Medical University 1838 Guangzhou North Road Guangzhou 510515

China

E-mail: qch.2009@163.com 